\title{
A Robust Multi-objective Desirability-based Simulated Annealing for Optimization of Sustainable Supply Chain Network Strategic Design
}

\author{
Kannan Govindan ${ }^{1,3}$, and Ahmad Jafarian ${ }^{2}$ \\ ${ }^{1}$ Department of Business and Economics, University of Southern Denmark, Odense, Denmark \\ ${ }^{2}$ Faculty of Management and Accounting, Allame Tabataba'i University Business School, Tehran, Iran \\ ${ }^{3}$ Institute of Applied Ecology, Chinese Academy of Sciences, Shenyang, China. \\ gov@sam.sdu.dk
}

\begin{abstract}
In this article, we investigate the optimization of sustainable supply chain network (SCN) strategic design problem. The proposed SCN is composed of four echelons including plants, distribution centers, cross-docks and retailers. The problem has been mathematically formulated as a multi-objective optimization model that aims to minimize the total costs and environmental effect. To tackle the addressed problem, multi-objective desirabilitybased simulated annealing algorithm (MODBSA) is proposed. Then, MODBSA is compared with two common meta-heuristic algorithms. Subsequently, the known method called response surface methodology with the multi-objective decision making(MODM) approach is employed to tune the parameters. Finally, MODBSA and other approaches are compared together in terms of some performance measures.

Index Terms - Supply chain network design, Sustainability, optimization, multi-objective desirability-based simulated annealing
\end{abstract}

\section{Introduction}

Supply chain network design (SCND) is considered as the most crucially important strategic decision in Supply chain management (SCM), and contains the determination of locations, numbers and capacities of network facilities and the material flow between them [1]. sustainable SCND (SCND) tries to define the best supply chain structure that enables an organization to maximize its long-term economic profitability and performance [2]. SCND models have traditionally focused on minimizing fixed and operating costs without taking environmental and social aspects into account [3]. Consequently, SCND is a new emerging approach that aims to consider the above mentioned three pillars of sustainability i.e., economic, environmental and social in the design phase[2]. The published papers about sustainable SCM are mainly focused on greenness and cost-effectiveness of networks. Only 4 out of 36 surveyed papers have been related to the social aspects as well as economic and environmental aspects [4]. However, these papers have only misused the term corporate social responsibility (CSR) while their models only have encompassed economic and environmental issues. In the other word, social impacts are inherently qualitative which cause the rareness of quantitative models in this dimension of SSCM.
Therefore, we are restrained to narrow down our literature review to green supply chain management. According to [5], the importance of environmental collaboration within a supply chain has been increasingly known. GSCM includes product shipment through the chain from plants to distributors to customers, and even reverse logistics [6]. Accordingly, our proposed model is focused on designing a forward GSCND. In terms of modeling approach and objectives, the most relevant work is [7], while in this paper, different levels of investments at plants with the aim of environment protection are considered.

In summary, the major distinguished innovations and features in this study are: (i) costs and environmental impacts of different technologies depend on potential plants' locations; (ii) a four-echelon SCN is modeled in which both direct and indirect shipments are allowed; (iii) contriving the desirability concept in MOSA and improving its performance. The rest of paper is as follow: Section 2 briefly delineates the problem description. Section 3 describes the definition of Pareto optimality and proposed algorithms. Finally, computational results and conclusion are presented respectively.

\section{Problem Description}

In this study, a single product forward supply chain by four echelons is considered, i.e. plants that different technology can be established on them, distribution centers that dispatch the products to a set of retailers via two different ways, direct shipment and cross-docks. Accordingly, in this model, SSCND is optimized by a two-stage mixed-integer programming model. In the first stage, decisions about choosing operationalized facilities from the potential facilities set (i.e. plants $(p \in P)$, distribution centers $(d \in D)$ and cross-docks $(c \in C))$, and technology $(t \in T)$ of plants are made prior to demands known, and in the second stage, decisions about the product delivery quantities. The notations used in the model are in follow: 


\begin{tabular}{|c|c|}
\hline Parameters & Description \\
\hline$T E C_{p t}$ & the acquisition cost of technology $t$ at plant $p$ \\
\hline$O C_{p}, O C_{d}, O C_{c}$ & the opening cost of facilities \\
\hline$V C_{d}, V C_{c}$ & the variable cost for handling products \\
\hline$M C_{p t}, E M_{p t}$ & the manufacturing cost and emissions of each unit product at plants, respectively \\
\hline$E T_{p d}, E T_{d c}, E T_{c r}, E T_{d r}$ & the per unit environmental impacts of transporting from facility $i$ to facility $j$ \\
\hline$E O_{p t}, E O_{d}, E O_{c}$ & the environmental impacts of opening facilities \\
\hline $\mathrm{Cap}_{p t}, \mathrm{Cap}_{d}, \mathrm{Cap}_{c}$ & the production/ handling capacity \\
\hline$P_{M a x}, D_{M a x}, C_{M a x}$ & the maximum desired number of facilities \\
\hline$T C_{p d}, T C_{d r}, T C_{d c}, T C_{c r}$ & the per unit cost of transporting from facility $i$ to facility $j$ \\
\hline $\operatorname{Dem}_{r}$ & the demand of $r \in R$ \\
\hline Decision variables & Description \\
\hline$\beta_{p t}$ & $\left\{\begin{array}{l}1, \text { if plant } p \in P \text { with technology } t \in T \text { is openned; } \\
0, \text { otherwise. }\end{array}\right.$ \\
\hline$\gamma_{d}$ & $\left\{\begin{array}{l}1, \text { if distribution center } d \in D \text { is opened; } \\
0, \text { otherwise. }\end{array}\right.$ \\
\hline$\lambda_{c}$ & $\left\{\begin{array}{l}1, \text { if cross-dock } c \in C \text { is opened; } \\
0, \text { otherwise. }\end{array}\right.$ \\
\hline$y_{r c}$ & $\left\{\begin{array}{l}1, \text { if retailer } r \in R \text { is assigned to cross-dock } c \in C ; \\
0, \text { otherwise. }\end{array}\right.$ \\
\hline$x_{p d}, x_{d c}, x_{c r}, x_{d r}$ & the flow of products from facility $i$ to facility $j$ \\
\hline$h_{p t}$ & the amount of product manufactured at $p \in P$ with $t \in T$ \\
\hline
\end{tabular}

\section{Mathematical Formulation}

$$
\begin{aligned}
& \text { Min } \mathrm{OBJ}_{1}=\sum_{p \in P} \sum_{t \in T}\left(O C_{p}+T E C_{p t}\right) \beta_{p t}+\sum_{d \in d} O C_{d} \gamma_{d} \\
& +\sum_{c \in C} O C_{c} \lambda_{c}+\sum_{p \in P} \sum_{t \in T} M C_{p t} h_{p t}+\sum_{p \in P} \sum_{d \in D}\left(V C_{d}+T C_{p d}\right) x_{p d} \\
& +\sum_{d \in D} \sum_{c \in C}\left(V C_{c}+T C_{d c}\right) x_{d c}+\sum_{c \in C} \sum_{r \in R} T C_{c r} x_{c r}+\sum_{d \in D} \sum_{r \in R} T C_{d r} x_{d r} \\
& \text { Min } \mathrm{OBJ}_{2}=\sum_{p \in P} \sum_{t \in T} E O_{p t} \beta_{p t}+\sum_{d \in D} E O_{d} \gamma_{d}+\sum_{c \in C} E O_{c} \lambda_{c} \\
& +\sum_{p \in P} \sum_{t \in T} E M_{p t} h_{p t}+\sum_{p \in P} \sum_{d \in D} E T_{p d} x_{p d}+\sum_{d \in D} \sum_{c \in C} E T_{d c} x_{d c} \\
& +\sum_{c \in C} \sum_{r \in R} E T_{c r} x_{c r}+\sum_{d \in D} \sum_{r \in R} E T_{d r} x_{d r}
\end{aligned}
$$

Subject to:

$$
\begin{aligned}
& \sum_{p \in P} x_{p d}=\sum_{c \in C} x_{d c}+\sum_{r \in R} x_{d r} \quad \forall d \in D \\
& \sum_{d \in D} x_{d c}=\sum_{r \in R} x_{c r} \quad \forall c \in C \\
& \sum_{d \in D} x_{d r}+\sum_{c \in C} x_{c r} \geq \operatorname{Dem}_{r} \quad \forall r \in R
\end{aligned}
$$

$\sum_{c \in C} y_{c r} \leq 1 \quad \forall r \in R$

$$
\beta_{p t}, \gamma_{d}, \lambda_{c}, y_{c r} \in\{0,1\} \forall t \in T, p \in P, d \in D, c \in C, r \in R
$$




$$
x_{p d}, x_{d c}, x_{c r}, x_{d r}, h_{p t} \geq 0 \forall t \in T, p \in P, d \in D, c \in C, r \in R
$$

The OBJ1 minimizes total variable and fixed costs. The first three terms are the fixed costs. While the above mentioned terms are associated with the first stage of model decided before knowing the demands the remaining terms are associated with the second stage. The fourth summation is the variable manufacturing costs at plants, and the rest terms represent the variable handling cost and variable transportation costs in the network. The OBJ2 is the environment objective. The first three terms of this objective represent the environmental impacts associated with opening facilities. The fourth summation is the environmental impacts produced by manufacturing at plants and the rest summations are environmental impacts related to shipping products thorough SCN. Eqs. (3\&4) guarantee the flow conservation. Constraint (5) ensures that the demand of each retailer is satisfied by a cross-dock and/or distribution center(s). Constraint(6) states that each retailer should be assigned at most to one cross-dock. Constraint(7) ensures that products are allowed to be sent from cross-dock $c$ to retailer $r$ only and only if the retailer $r$ is assigned to cross-dock $c$. Eq. (8) should be satisfied to compute the amount of products which have to be manufactured in plant $p$ under technology $t$. Constraints (9to11) ensure that products could be shipped only from opened facilities. Constrain $t(12)$ ensures that in each potential node for plants at most one plant with only one technology is established. Inequalities (13to15) limit the maximum number of opened facilities. Finally, all variable are defined in lines (16\&17).

\section{Multi-Objective Optimization Problem}

To solve this multi-objective optimization (MOO) problem, the Pareto optimal method based on non-dominance concept is utilized. More definitions of Pareto optimality can be found in [8]. Also, it is said that solution $x$ dominates solution $y(\vec{x} \prec \vec{y})$, i.e. $f_{k}(\vec{x}) \leq f_{k}(\vec{y}) \forall k \in\{1,2, \ldots, K\}$.

\section{Multi-Objective Algorithms}

Regarding solution methods, we have proposed multiobjective desirability-based simulated annealing (MODBSA). Furthermore, to examine the proposed method, some experiments are designed and conducted in which, the results of proposed algorithm is compared to NSGAII as one of the best multi-objective genetic algorithms based on methods[9] and multi-objective particle swarm optimization algorithms (MOPSO) as one popular swarm intelligent algorithm [10].

\section{MODBSA}

Simulated Annealing(SA) is one of the stochastic search algorithms, which was popularized by [11]. Due to its theoretical guarantee of convergence and ease of implementation, it has been known and widely applied for many optimization real-world problems [12]. The first multiobjective SA (MOSA) was proposed by [13]. As, a critical obstacle for MOSA can be known its inability to find multiple solutions, we propose the population-based MODBSA with both intensification and diversification. This method, after initialization stage, a neighbor around the initial solution will be found, and this neighborhood search (NS) is repeated $\operatorname{Max}_{n M}$ times. The new neighbor solution $\left(x_{\text {pop,nMove }}\right)$ is compared with the best solution $\left(x_{\text {pop best }}\right)$ that is acquired for that particle, and if $x_{\text {pop,nMove }}$ dominates $x_{\text {pop best }}$, the new solution will be accepted, but if $x_{p o p, b e s t} \prec x_{p o p, n M o v e}$, the new solution will be accepted with transition probability. The transition probability from state $i$ to $j, P_{t}(i, j)$ with random cost criterion (there are a variety of criteria e.g. minimum cost, maximum cost, random cost, self cost, average cost and fixed cost [14]), While in this paper, the desirability concept is utilized as the most suitable policy for this approach, that is calculated as:

$$
P_{t}(i, j)=\min \left\{\exp \left(-\frac{D_{j}-D_{i}}{T_{j}}\right), 0\right\}
$$

Where, $D$ is desirability of each solution in comparison with ideal solution $\vec{f}_{\text {ideal }}=\left\{\min f_{1}, \ldots, \min f_{k}\right\} \quad$ and $\vec{f} \in\left\{X_{1, \text { best }}, \ldots, X_{n \text { Pop p best }}\right\}, T_{j}$ is the annealing temperature, $D_{i}=\sqrt[k]{d_{i, 1} \times d_{i, 2} \times \ldots \times d_{i, k}}, k$ is the number of objective function and $d_{i}$ is calculated as[15]:

$$
d_{i, k}=H_{k}-f_{i, k} / H_{k}-L_{k}
$$

Where $\vec{L}=\left\{\min f_{1}, \ldots, \min f_{k}\right\}, \vec{H}=\left\{\operatorname{Max} f_{1}, \ldots, \operatorname{Max} f_{k}\right\}$ and $\vec{f} \in\left\{X_{1, b e s}, \ldots, X_{n p o p, b e s}\right\}$. Finally, if $x_{p o p, n M o v e}$ and $x_{p o p, b e s t}$ do not dominate each other, one of them selected randomly. By using this trait, if the best solution is not improved, the algorithm is guided to another region and perhaps finds a better solution. The proposed algorithm is guided by the initial temperature $T_{0}$, and cooling rate $T_{f}$, these values essentially dictate the acceptability of solutions found during the search. The temperature reduction form used in MODBSA is $T_{j+1}=\alpha^{*} T_{j}$ where $\alpha=\left(T_{f} / T_{0}\right)^{\wedge}(1 /$ MaxlsItr $)$. In each iteration, if the number of $\tilde{A}$, exceed than $n P o p$, the $n P o p$ member of $\tilde{A}$ is selected based on crowding distance [9]. The quality of obtained responses significantly depends on the efficiency of the developed NSs. In this paper, various types of NS structures are used to generate a new solution.

\section{Computational Results}

To compare the proposed algorithm (MODBSA) with other algorithms (NSGA-II and MOPSO), the comparisons are performed on the basis of the sets of solutions obtained by each algorithm.

\section{Data Generation}

In order to compare the proposed algorithm with other benchmark algorithms, an experiment was conducted to 
deliberate on the performance of different metaheuristics. Considering the numbers of potential units in each echelon the problems are classified into three scales (Small, medium, large). In each scale, eight random problems are produced.

\section{Evaluation Metric}

In order to compare the quality of different Paretooptimal fronts produced by MOO methods, and due to the conflicting and incommensurable nature of them, various performance assessment metrics are used, simultaneously. In this paper, the following metrics are used:

- Diversification metric (DM): specifies the diversity of solutions came by algorithms. Refer to [16].

- Mean ideal distance (MID): The nearness among the Pareto solutions and ideal point $(0,0)$. Percent of Domination (POD): In order to calculate this metric, among all non-dominated solutions acquired by the algorithms, a combination Pareto set is constructed then the percentage of the solution belonging to each algorithm is calculated. For this performance measure, the algorithm with higher value has better performance.

Table 2. Tuned parameters, $R$-squared $\left(\mathrm{R}^{2}\right)$ and Desirability (D)

\begin{tabular}{|l|l|l|l|l|}
\hline & Tuned parameters & $\begin{array}{l}\mathbf{R}^{\mathbf{2}} \\
\text { Diversity }\end{array}$ & $\begin{array}{l}\mathbf{R}^{\mathbf{2}} \\
\text { SNS }\end{array}$ & $\begin{array}{l}\mathbf{R}^{\mathbf{2}} \\
\text { POD }\end{array}$ \\
\hline NRGA & MaxIt $=596, P_{c}=0.758, P_{m}=0.4$ & $76 \%$ & $72 \%$ & $82 \%$ \\
\hline NSGAII & MaxIt $=600, P_{c}=0.796, P_{m}=0.36$ & $72 \%$ & $84 \%$ & $80 \%$ \\
\hline MOPSO & MaxIt $=568, n P o p=80, n$ Grid $=12$ & $58 \%$ & $61 \%$ & $69 \%$ \\
\hline MODBSA & MaxIt $=584, n P o p=86, n$ Move $=8$ & $62 \%$ & $58 \%$ & $68 \%$ \\
\hline$P_{c}$ (probability of crossover) and $P_{m}$ (probability of mutation) \\
\hline
\end{tabular}

\section{Parameters Setting}

Parameter setting is identified as a crucially important milestone to achieve robust metaheuristic algorithms by not producing functional variance under external environment influence. This paper employs the Response Surface Method (RSM) as a well-known technique that is widely applied in parameter optimizations, to determine the optimal values of the parameters. The calculation is done based on [18]. To compare the algorithms fairly, the population of nondominated frontiers was set constant, (i.e. nPop in NSGAII and MOPBSA, nRep in MOPSO is considered 50). As some performance assessment metrics is used together for making quantitative comparisons in multi-objective metaheuristics, a selection problem is introduced to set input factor's value based on represented performance metrics simultaneously, which leads to the desired response in multi-objective decision-making (MODM) environments. The tuned values for estimated parameters and R-Squreds are displayed in Table 2.

\section{Comparisons among Algorithms}

In order to determine whether there is a significant difference among the performance of algorithms, after the calculation of Pareto optimal solutions for all three algorithms for each problem, the efficiency of each algorithm is assessed in terms of DM, SNS and POD. To find out the best algorithm decisively, a statistical comparison among the algorithms in each performance measure is done. In this regard, first, the results obtained for each problem are transformed to Relative percentage deviation(RPD)[17]. Then, Kruskal-Wallis analysis of variance (ANOVA) that is a non-parametric method for testing whether samples originate from the same distribution, have been performed. The obtained results verify that at least two algorithms are not identical in two metrics (DM: $H=12.32, P=0.001 *$; MID: $H=5.23, P=0.06$; POD: $\left.H=24.6, P=0.00^{*}\right)$. Also, Fig. 1 indicated in SNS and POD metrics, MODBSA significantly is better than the other algorithms. Also, it can be declared that with the growth of the dimensions of the problem, the efficiency of the proposed algorithm outperforms other approaches. In MID, there is not any significant hegemony among the algorithms.
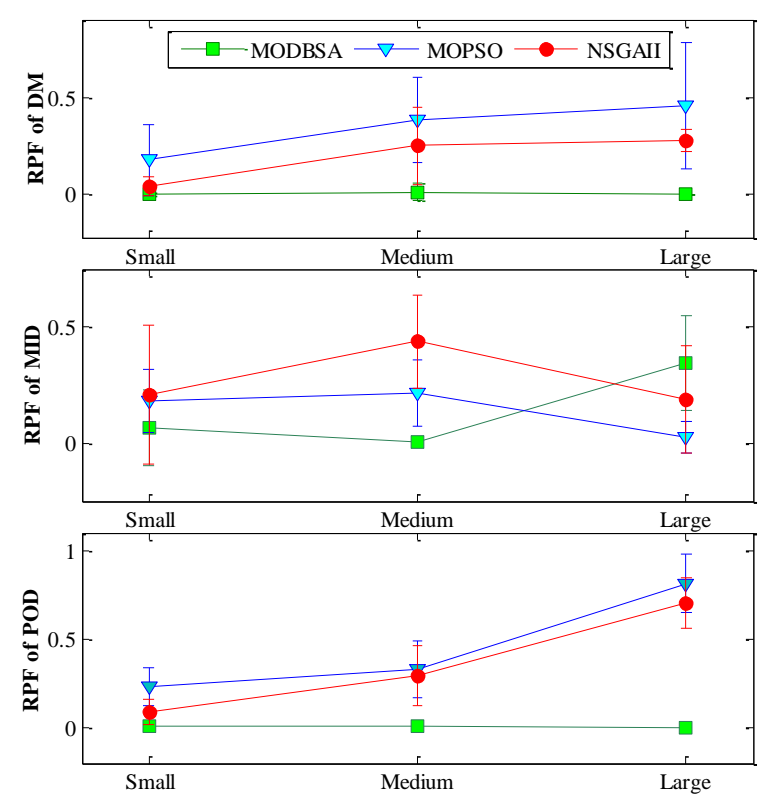

Figure 1. Evaluation metric value for the interaction between type of algorithm \& size of problems

\section{Conclusions and Future Works}

In this paper, we proposed multi-objective desirabilitybased simulated annealing (MODBSA) approach to strategically design a multi-objective sustainable SCN with the aim of minimizing the total costs and environmental effects, simultaneously. To validate the proposed algorithm, 24 test problems are produced in three size (small, medium and large), and the performance and the reliability of the proposed algorithm were evaluated in comparison with three popular methods (i.e. NRGA, NSGAII and MOPSO). To have a fairly comparison, the algorithms were compared in a robust mode, due to this, all the algorithms' parameters were estimated by RSM and MODM approaches based on four different 
comparison metrics were utilized to validate the efficiency of the algorithms. The results obtained by the proposed algorithm almost dominate the results of other approaches. Finally, the sensitivity analysis for the performance of algorithms demonstrated that MODBSA yielded better solutions in comparison with NRGA, NSGAII and MOPSO. For further research, other or newer strategies of meta-heuristic methods are suggested to obtain a better solution on this problem. Finally, the future studies can develop the society aspect of sustainability in SCND.

\section{Acknowledgements}

This study was supported by Natural Science Foundation of China (71033004), Chinese Academy of Sciences (2008$318)$ and Ministry of Science and Technology ((2011BAJ06B01).

\section{References}

[1] M. S. Pishvaee and J. Razmi, "Environmental supply chain network design using multi-objective fuzzy mathematical programming," Applied Mathematical Modelling, vol.36(8): pp. 3433-3446. 2012.

[2] A.Chaabane, A. Ramudhin, and M. Paquet, "Design of sustainable supply chains under the emission trading scheme," International Journal of Production Economics, vol.135(1): pp. 37-49. 2012.

[3] S. Elhedhli and R. Merrick, "Green supply chain network design to reduce carbon emissions," Transportation Research Part D: Transport and Environment, vol.17(5): pp. 370-379. 2012.

[4] J.M. Cruz, "The impact of corporate social responsibility in supply chain management: Multicriteria decision-making," Decision Support Systems, vol.48(1): pp. 224-236. 2009.

[5] S. Vachon and R.D. Klassen, "Environmental management and manufacturing performance: The role of collaboration in the supply chain," International Journal of Production Economics, vol.111(2): pp. 299-315. 2008. 